\title{
Mobile ascending aortic mass presenting as acute myocardial infarction
}

\author{
Hellmuth R. Muller Moran, MD • Calvin Loewen, MD • Vikas Dutta, MD • \\ Pallav J. Shah, MD
}

Received: 10 December 2017/ Accepted: 2 January 2018/Published online: 11 January 2018

(C) Canadian Anesthesiologists' Society 2018

A 56-yr-old (62 kg) man (who provided written consent to publish this report) with no documented medical history presented to the emergency department after eight hours of

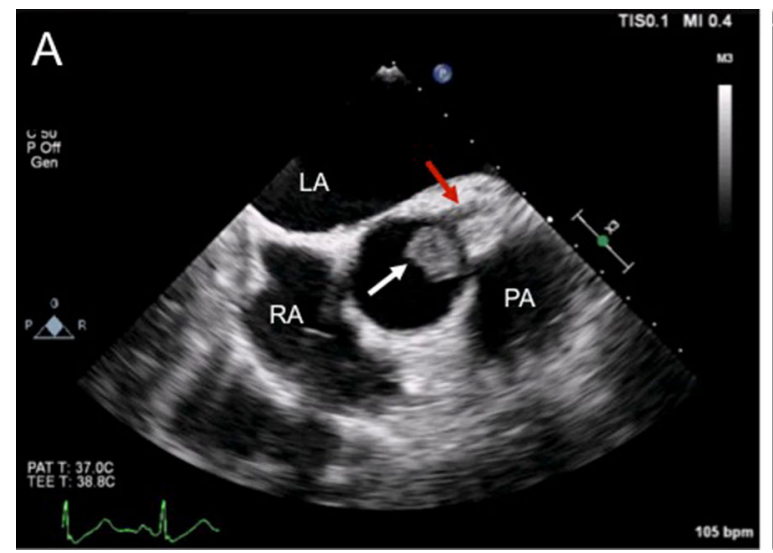

Figure A) Two-dimensional transesophageal echocardiographic (TEE) short-axis image of the aortic root shows a thrombotic mass (white arrow) close to the the left main coronary artery (red arrow) ostium. B) Three-dimensional TEE long-axis image similarly shows

Electronic supplementary material The online version of this article (https://doi.org/10.1007/s12630-018-1052-z) contains supplementary material, which is available to authorized users.

H. R. Muller Moran, MD · P. J. Shah, MD ( $\varangle)$

Department of Surgery, Max Rady College of Medicine,

University of Manitoba, Winnipeg, MB, Canada

e-mail: pallavshah@ozemail.com.au

C. Loewen, MD · V. Dutta, MD

Department of Anesthesia \& Perioperative Medicine, Max Rady College of Medicine, University of Manitoba, Winnipeg, MB,

Canada chest pain. With a normal electrocardiogram and a troponin $\mathrm{T}$ level of $2720 \mathrm{ng} \cdot \mathrm{L}^{-1}$ (normal range $0-14 \mathrm{ng} \cdot \mathrm{L}^{-1}$ ), he was subsequently diagnosed with a non-ST segment elevation

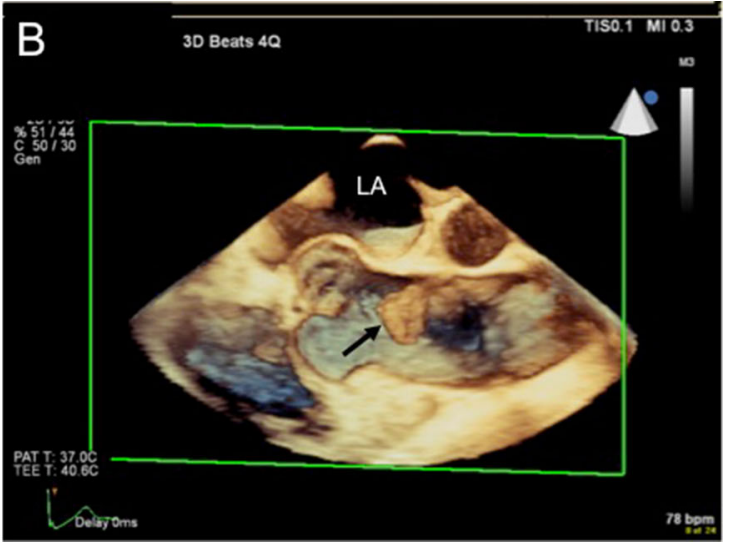

the mass (black arrow) in the ascending aorta. A video of the mobile mass is available as Electronic Supplementary Material. LA = left atrium; PA = pulmonary artery; $\mathrm{RA}=$ right atrium

myocardial infarction. Transthoracic echocardiography unexpectedly revealed a $1.3 \times 1.7 \mathrm{~cm}$ mobile mass in the ascending aorta close to the left main coronary ostium.

He was scheduled for urgent resection of the mass under cardiopulmonary bypass (CPB), at which time transesophageal echocardiographic examination further characterized the mass (Figure A and B). It appeared to be encapsulated with a very small stalk of attachment immediately adjacent to the left main coronary ostium. The mass was resected en bloc, with the associated aortic wall removed by shave excision. The aorta was closed, and the 
patient was weaned from $\mathrm{CPB}$ and transferred to the intensive care unit.

As postoperative coronary angiography did not reveal any obstructive coronary artery disease, the patient's initial presentation was thought to be due to either embolization or transient occlusion of the left main coronary artery. He was discharged home on postoperative day 7 after an uneventful course in hospital.

Pathological examination revealed that the mass was composed entirely of thrombus, with portions showing evidence of hyaline degeneration, suggesting that the mass had been present for quite some time. As no bacterial or fungal organisms were isolated, and the patient had no known hypercoagulability disorder or atherosclerotic disease, the source of the thrombus remained unclear.

Acknowledgments The authors thank Dr. Eric Jacobsohn and Dr. Hilary P. Grocott for their assistance in preparing this publication.

Conflicts of interest The authors declare that there are no conflicts of interest related to this work.

Editorial responsibility This submission was handled by Dr. Philip M. Jones, Associate Editor, Canadian Journal of Anesthesia.

Funding No funding was used to conduct this work. 